\title{
Curties notes llingüístiques a un testu castellán del sieglu XVIII
}

\author{
X.Ll. García Arias
}

L'asoleyamientu de la obra de 1712-1714 del dominicu asturianu fray Toribio de Santo Tomás y Pumarada, Arte General de Grangerías ${ }^{1}$, ye un puntu de referencia de bona importancia p'afondar daqué na conocencia de la llingua asturiana nos sieglos XVII-XVIII. N'efeutu, como fexeron ver los responsables de la edición, nella atópase un bon verbeneru de datos léxicos lo qu'aconseyó inxerir a lo cabero la obra un glosariu d'unes seiscientes pallabres asturianes. Como nós mesmos alvertimos llueu (Lletres Asturianes 93: 169) númberu talu habría allargase enforma pues una llectura más posada dexa ver aspeutos onde ye posible, magar les torgues del continuum asturianu-castellán, facer una dixebra llingüística más curiosa ente los dominios. Foi asina como arriquecimos abondo la información léxica, a vegaes fixándonos en dellos calcos semánticos, que yá s'afaya ente los materiales documentaos del nuesu Diccionariu Etimolóxicu de la Llingua Asturiana (iguándose).

Al lleer con procuru la obra de Pumarada, naide nun dexará d'encontar esta suxerencia perviendo cómo a lo llargo de les más de milenta páxines de prosa castellana, a vegaes trupa, dacuando apolazada y llixera, van entremeciéndose abondos términos y bayuroses construcciones gramaticales que, si bien dacuando podría discutise si respuenden a un usu xeneralizáu del castellán d'aquella dómina o a un arcaísmu meditáu del so autor, delles nun sedríamos a xustificar

1 Fray Toribio de Santo Tomás y Pumarada (2006): Arte General de Grangerías (1712-1714). I: De la grangería espiritual. II. De las grangerías temporales. Salamanca (ed. San Esteban \& Muséu del Pueblu d’Asturies), 2006. Ed. y estudiu preliminar de Juaco López.

CES.XVIII, núm. 16 (2006), págs. 77-88. 
si nun fóramos sabedores del aniciu asturianu del so responsable, natural de Colunga na fastera centro-llateral del dominiu llingüísticu ástur².

La presencia direuta del asturianu pervése nel nivel fónicu-fonolóxicu unes vegaes gracies al préstamu plenu y otres a l'adautación del asturianismu, como alvertimos acabante ponenos a lleer el glosariu. Pero tamién resulta d'interés exemplificar l'asturianismu llingüísticu de la obra amosando dellos de los trazos de tipu morfolóxicu y sintáuticu más presentes nes xeneroses páxines d’esti yá

2 Ello nun desanicia de la prosa de Pumarada dellos usos bien alloñaos non sólo de la norma castellana sinón de los vezos llingüísticos asturianos y que respuenden, de xuru, al influxu popular de Castiya onde'l nuesu autor desendolcó bona parte del so llabor relixosu. D’ehí una presencia abondosa del leísmu y del laísmu de lo qu'ufiertamos una esbilla. Tamién ta presente el loísmu pero, acordies col espardimientu dialeutal del mesmu, mui curtiamente:

leísmu plenu

Si es persona casada (...) le debo atender (...) y tenerle propiamente por mi mayor, y honrrarle como a tal [251]

hasta ponerles $\{$ a los viajeros $\}$ en segura vereda [205]

\{a los superiores $\}$ deben honrrarlos a ellos, inferiores. Y por consiguiente deben amarlos (...) / Deben obedecerles, reverenciarles y socorrerles [251]

serás burro en no proponérsele [611]

$\underline{\text { le }}$ debes $\{$ al potro\} guardar para el oficio [892]

y prenderle \{al castrón\} [915]

\{el carretaje\} hazle tú con tus bueyes [695]

este pecado de estupro le comete [339]

aunque pedido \{el débito conyugal $\underline{\text { le }}$ debe dar [342]

hasta oy le han habitado \{el mundo\} [349]

regadío, si le ay [566]

Mi secreto para mí no para decirle a tí [201]

el grano temprano si le siembras [808]

el frío quando le hace [849], etc.

laísmu

Y el señor que la dio algo para el primer matrimonio [217]

sino dezirla \{a la mujer\} con flema [249]

ni sea fácil en creerla \{a la mujer\} quanto diga [249]

quitarla la integridad física [340]

si para que dentro descargue la dize cariños [348]

\{esas mujeres\} son buenas para (...) quien no tenga que las mandar [216]

lo uno dándolas tu lección \{a las hijas\} [223]

por dilatar sus padres darlas marido [227]

hacerlas unas madreñicas ligeras [553], etc.

loísmu

los tutean, los demenan, $\underline{\text { los }}$ dizen quantas llanezas se les vienen a la boca (...) los dizen cien sobarbadas [229].

Otros fenómenos como determinaos usos de deísmu, sedríen entendibles, quiciabes, dende les posibilidaes evolutives del cast. y del asturianu:

nunca pensó de recibir semejante alegría [79], necessita de hablar [334]

necessitan de ajunterarse [679], no necessitas de sembrar la grana [1005], etc. 
emblemáticu testu del nuesu sieglu XVIII llaráu d’interferencies pue qu’unes vegaes conscientes, pue que dacuando inalvertíes ${ }^{3}$.

\section{Aspeutos fónicos y fonolóxicos}

1. Diptongación acordies col asturianu:

banielles, cibiella, enrriéstranse, estiella/estilla, formiento, forniella, biesca, piesco, tiez, etc.

fueya, bueyo $\rightarrow$ *abueyar $\rightarrow$ aboyar

2. Presencia eventual de $-u$ anque lo normal ye xeneralizar con -o los préstamos asturianos masculinos:

sayu, fariellu/fariello, corripio, coladorio, corripio, corteyo, cribo, cucho, desco, llombo, escobayo, estoyo, estro, fecho, felpeyo, fierro, furaco, gocho, etc.

3. Presencia de $a$ - protética especialmente na formación de verbos:

arralar, arrañar, arromper, arreyar, etc.

4. Presencia de [j] epentética: corripio, murio, oricios, etc.

5. Caltenimientu de -e nun exemplu como rede

6. Inseguranza nel vocalismu átonu: embernar, embeligu, reñón, semiente, nuzal, etc.

7. Presencia perfrecuente de F-: afondar, afornellar, aforrar, afuracar, faba, fame, fariellu, farina, faya, faza, faza, faya, felencho, fender, fossoria, etc.

Mesmamente cuando se trata d'una -FF- : mofo (cast. musgo)

Atópense dellos exemplos con aspiración de $f$ - que nun sabríemos dixebrar si respuende a un influxu asturianu oriental o a restos castellanos de la vieya aspiración enantes de la so perda xeneralizada: jatados 'rebaños'

8. Palatalización perfrecuente de $\mathrm{L}^{-}>[\lambda]$ : llabrar, llaceria, llaciada, lladral, llagar, llegra, etc.

9. Palatalización de LJ, C', G'L > [y]: aguyeta, amiyar, arbeyos, ayo, boruyo, caleya, corneyal, coyer, cuyar, dayuri, estadiya, estorneya, folleya, mayar, oveya, paya, etc.

3 Nun sabríamos si nel asitiamientu del nuesu autor pue vese una vía abierta onde naguar por un modelu que perpase lo estrictamente castellano, daqué na llinia teórica que Fernando González-Ollé alvierte en dellos autores del sieglu XVIII: «La dialectologización de Castilla la Vieja en el siglo XVIII». Anuario de Letras XXIX. (Homenaje a Dámaso Alonso). México, Facultad de Filosofía y Letras, \& Centro de Lingüística Hispánica.

Per otru llau gráficamente, amás de los problemes en rellación dellos resultaos palatales o velares (\&11), nun se plantega mayor problema mesmamente nos casos alloñaos güei de la norma como ye escribir «s» 0 «ss»

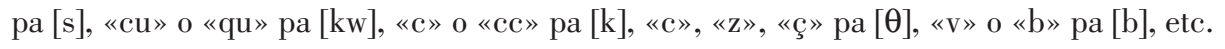


10. Palatalización de DJ: estaya, estoyo, etc.

11. ¿Palatalización o velarización?

La conducta llingüística de Pumarada, qu'unes vegaes ufierta l'asturianismu crudu y otres adauta dafechu al castellán, fai qu'en delles ocasiones duldemos de cuál de les víes escueye na so escritura. Esto pasa particularmente a vegaes que nun sabemos si nagua por escribir una palatal asturiana [̌̌ $]$ o la so correspondiente castellana $[\mathrm{x}]$. La grafía «x» nesi sen nun esclaria un res la dulda darréu que dacuando «x» (asina en exa) debemos entendela a l'asturiana, y más si como nel casu citáu otros elementos morfolóxicos lo favorececen. Cuando la grafía ye «j», «ge, «gi», pasa lo mesmo pues si ye verdá que ye tamién grafía de [x] dacuando camentamos que podría emplegase cenciellamente pal asturianu [̌̌s] como en juncla 'xuncla', junclo 'xunclu', corejina 'corexina', jata 'xata', jugo 'xugu', etc. En dellos de casos esa dulda asítiase tamién cuando'l resultáu asturianu esixiría [y] como en mes de pajares 'mes de payares, noviembre', paja 'paya', etc.

12. Tratamientu de N-, -NN-:

Nun hai nicios de palatalización de N- anque exemplos como niero 'ñeru, nial' podríen plantegar la dulda de si se trata d'una grafía de [ñ], lo mesmo que vemos en anear, güei añar 'acunar', 'arrollar al neñu'. Continuadores de la xeminada dulden ente los resultaos palatales y los non palatales de lo qu'entá hai nicios nestos díes na fala de Colunga:

boroña/borona, cabañicas/cabanín, penayo/peñoso, peñarar/vanar

13. Presencia de -MB-:

embelga, llamber, llombo, palombina, etc.

14. Tratamientu de -M'N-:

Al llau del resultáu xeneral na fastera centro-occidental d'Asturies (brima, ensamar, fame) hai nicios d'una confluencia col castellán o, cenciellamente, castellanismos (combral, fembra, etc.).

15. Dellos exemplos de nasal epentética: monjeca 'moxeca', ponja 'poxa'.

16. Velarización del primer elementu de los grupos consonánticos romances: caltener, esllelda, llaldar, pielga, etc.

17. Dalguna confusión de líquida agrupada: clin.

\section{Aspeutos morfosintáuticos}

1. Nicios del femenín plural -ạs $>-\underline{e s}$ cádabes [673] , brimes [681], alberques [735], cotolles [787], gabielles [793], las gabielles [794], fabes [853], coricies [857] / corizas [865],

4 La cifra ente corchetes entiéndese como páxina de la edición. 
estas perendengues [884], colas / coles [988], fabes prietes [994], juncles [1030], nidios como llontres [830], para engordar como llontres los bueyes [276], etc.

2. Destremación de xéneru nel nome:

el labor [218], el dote [219], la canal [565], las zereçales [630], las castañares [630], este sal [829] / la sal [846], cobil mullida [921], la cubil [922], el techumbre [957] / la techumbre [956], etc.

3. Posesivos:

Caltién delles veces, en 3. ${ }^{\text {a }}$ persona, la espresión asturiana:

y lo mandó so merced [270], tus muchachas siempre a so man [194], echando so cuerno [630], cortar a so pode [660], etc.

Pero la espresión ye castellana dafechu nel restu de los casos anque pue almitir posesivos con anteposición del artículu, d'usu xeneral n'asturianu anque posible dialeutalmente en castellán:

los mis tíos y tías [76], en el tu lugar [227], en la tu Riera [521], el tu pidial [602], etc.

4. Indefiníos:

Los usos asturianos paecen claros nel emplegu indefiníu de daqué, qu'entá se caltién en delles fasteres del dominiu:

si es daqué tanto [866]

siendo \{tus ovejas\} daqué tantas [907]

Tamién exemplos de ál que respuenden al asturianu de güei, pelo menos como pronome, nel oriente del país (DGLA) pero yá arcaicu nel castellán del sieglu XVI (DCECH s.v. al); la documentación del testu que comentamos fai ver tamién un emplegu axetivu:

en parte retirada de lo ál gente [242]

todo lo ál del medio [723]

para la guarda de lo ál ganado [854]

lo ál cierro a las beras de las riegas será facilíssimo de perpetuar [744] basta lo ál de las primeras tremerías [748]

5. Allugamientu de clíticos:

porque estos señores, no les pidiendo dineros [215]

\{esas mujeres\} son buenas para un tendero o quien no tenga que las mandar [216]

no se lo dar demasiado a entender [250]

¿...y no los poder ver más que sangre en pan? [261]

por no se ver encerrados [536]

no les dar esse gusto [564]

no te aprovechar [745] 
de no lo hacer assí [931]

el no los tener occiosos [942]

después irlo espadando [818], etc.

6. Haber por facer

como si huviera treinta años [769] 'fexera'

quando ay el calor [695]

quando piensa que pilla una yegua de ai ocho años, hallarse después en cassa con yegua de doze años que es gran burla [871]

7. Desaniciu d'artículu na amestanza en + masculín:

cocidas en caldero [605], en desbán [625], acaba en seis de marzo [658], etc.

8. Amestanza en $+\underline{\text { xerundiu }}$, xeneral güei n'asturianu y posible nel castellán d'otres dómines:

en faltando el cuidado [516], en levantando por abril el sol [783],

en acabando [802], en teniendo doze años [883], etc.

9. Doble preposición:

Anque posible la so documentación en testos castellanos l'usu caltiénlu con puxu l'asturianu güei:

para en cassa [227], para en costeras [705], para en el suelo [1127]

para en esse lugar [531], por entre las porretas [1068]

de a fecho [530], reales de a ocho [604], etc.

10. Perífrasis:

salir fuera (...) a fogueras [193], andar a payos, magüestos, furtos de fruta y molinos [193], cada res anda a comer [938], que yo estoy ha enseñarte [531], etc.

11. Perda de la preposición de:

Dacuando ye fácil alvertilo:

* puntica de madera $\rightarrow$ la dicha puntica madera [596]

De toes maneres non siempre resulta amañoso velo darréu qu'en munchos exemplos nun se ye a saber ú se trata d'una axetivación, d'una numberación tapecida pola ausencia de puntuación, o d'una perda de la preposición de:

*la faza de lagar $\rightarrow$ la faza lagar [675],

*la faza de bodega $\rightarrow$ la faza bodega [676]

*del piso de lagar $\rightarrow$ del piso lagar [678]

*un gran coz de roza $\rightarrow$ acullá un gran coz roza [1133]

*tuquetos, samocos $\leftarrow$ tuquetos samocos [686]

*tenadas, pajares $\leftarrow$ tenadas pajares [795], etc.

A la posibilidá de desaniciu de la preposición de sigue darréu la inseguranza de delles construcciones con haber: 
has de poner tienda / has trabajar con cuidado [517]

se han sembrar aí [576], el potro que avías capar [894],

12. Prefixu de superlativo: perllenar [637], perplantar [645], percubiertas [647], perzafarle [771], etc.

13. Sufixos:

diminutivos:

cubico [528], cabañicas [533], llanecico [535] llosica [535], canalica [566], caminicos [569], callandico [529], tuericos [585]

pezuelos [625], peñayuelo [633], plazuela [649], gabituelo [650], caleyuela [738], regachuela [749], pastorçuelo [939]

tuqueto [578], paleto [616], forquetas [620], verdialetos [620], viguetas [725], banquetos [903], maderetos [933], puertetas [934]

achete [578], cordelete [592]

fisgo/fisguito [643], pasera [661] $\rightarrow$ paserita [660], gabito [662], desquitos [935]

Diminutivos en -ín/-inos:

Frente a tarines [84], escarpines [553]:

javalinos [525], avellanos montesinos [685], artos montesinos [729], tapinos [724], andrinos [729], copinos [829], chorrinos [835],

remelguinos [874], chibinos [910], alfolinos [944],

gusaninos chiquinos [1042], menudín [571, 884], furaquinos pequeñinos [572], desquina [572], peruyalinas [573], pebidinas [579],

folleyina [596], campanín [830], clavelucos menudinos [1008], etc. despeutivos:

señoraco [529], pradacos [543], riegacos [566], trapacos [590], maderacas [664], racimos verdacos [699], barganacos [673], bacuca [768]

aumentativos:

bergamotas [600], varotas [738], vigotas [742], este vigote [742], maderote [742], filote [593]

furtones [655], gabitones [668], pontones [670], tarabicona [710], pertegones [730], fazona [734], caleyona [735], pontón [738], los vigones [739], llabiegón [771], argomonas [655]

peyorativu:

verllatos [572], verllaticos [590], herbatos [625]

instrumental:

barredorio [657], coladorio [700], cobertorio [709], bassorio [767], sechorio [771], estandorios [774]

abondativos, coleutivos:

llagaradas [701], pontonada [738], narvassada [828], cabrada [911] 
tu molinada [1003], molendada [1088], follicados [1087]

castañal, zerezal, guindal, etc.

arbolío [518], ralío [571], sequíos [782], baquío [524], fembrío [940], arboledos 524], etc.

otros:

andadiça [710], fechiças [724], raigaños [730], cestaño [1016], obrerudas [656], cabezorra [656], verdascas [668], muriego [711], etc.

doble sufixu:

gentecica [542], veriquina [566], faldequina [747], llombequín [724], pontoneto, crías belladillas [834], furaquinos [572], furaquitos [911], fondrigoncico [747], etc.

14. Axetivación güei almisible n'asturianu pero non en castellán:

la ruin res [860], con ruin ganado [860], ruines pucheros y más ruines crías [861], ruin toro [861], fembras ruines [861], jatos ruines [862], ruin ganado [576], ruin substancia [6000], ruines mudas [584], ruines tribas [600], ruin casta [843], ruina canalla [845], ruin bacca [845], crías \{de las vacas\} ruines y afeminadas [845]

guapa cinta [551], árboles guapos [590], guapa bodega [681], perro guapo [851],

Non siempre ye fácil alvidrar si se ta delantre d'usos con raigañu popular o si se trata d'una ayalga estilítisca del escritor, anque quiciabes l'orde axetivu + nome nos empobine a la segunda de les posibilidaes:

de sus nidias cañas [606], un nidio paleto [616] / maderas nidias [609]

loco prado [783], famiento maíz [791], bello pan [809], bello grano [813]

a tus regalados bueyes [829], sonantes lluecas [867], ponerlas \{a las yeguas\} galanas de clin [877], etc.

Per otru llau axetivos güei inesperaos, como tierra amorosa [996], magar les apariencies llévennos claramente a la primera de les opciones darréu qu'entá ye d'usu espresión tala n'asturianu.

16. Nominalización:

castañares pollas [633] / baxa a las pollas de Pedro Martín [632], solas pollonas castañares [751] / dichas dos pollonas [751]

vino chacolín [699] /el chacolin [699]

beras befales de los ríos [1001] / un befal para heredad [537], etc.

17. Posibles deverbales:

Xeneralmente en $-e$ :

a pode [587], este pode [661], el pode [661], un pode [1059] 
entallar $\rightarrow$ entalle [662], ésta muy al juste [691], haciendo abanze del sal [943], al rape, etc.

Pero ye posible un intentu percaracterizador en $-a,-o$ acordies con tendencies presentes na llingua:

al rapa [587], no haya rebalsa de agua con las lluvias [807], se ha dexar llenar de remansa [816], para mierca [836], en la tosquila [908], día de escolmena [1050], etc.

el broto [611], brotos [599], el pisso de las ubas [700], la voz será tu ganancia a medida del pido [807], tal pruido [847], el abanzo de la leche [852], etc.

En dalguna ocasión la nominalización pue facese sobro un participiu fuerte:

con este cuido [975] (frente a cuidado), la ración de harina y paja y otras veces la de alcacer con sego [841] (frente al ast. segáu)

18. Concordancia con non-cuntables:

Acordies cola conducta de la variante centro-oriental de la llingua obsérvense concordancies inequívoques:

los criarás al pie del mismo ganado, y que con ello duerma [854]

muy listos \{los perros\} delante de ello \{el ganado\} [858]

Ello la gente galana a todos parece bien [216]

quálo de las dichas tierras es pintado para prado y quálo mejor para heredad [779]

Tamién nesti sen s'atopen construcciones como yerba menudín [850] propio de la fala de Colunga y oriente frente al usu castellán yerba algo más gruessa [850]. Otres vegaes la solución escoyida foi la variante central quiciabes fuxendo de la oriental muncho más llamativa con -u:

pulpa abondo [643], yerba abondo [877]

19. Lo valle ¿nome con artículu neutru?:

Quiciabes l'usu más «anómalu» en rellación cola norma que se podría esixir al testu castellán de Pumarada taría na presencia del artículu lo antepuestu a un nome. Trátase d'una llista curtia d'exemplos como:

(1) la cassa se ha de hacer en lo valle cimero [735]

(2) en medio de lo valle hacia El Espineral [755]

(3) con robles en lo valle y a la falda de baxo [764]

(4) Y todo lo valle apacible de riega en medio será una gran vega de pradería [1130].

Al nuesu entender estos usos con lo nun sedríen a xustificase como arcaísmu de la vieya espresión del artículu masculín que convive con $e l$ na documen- 
tación ${ }^{5}$ y del que queden nicios del tipu lo bilugu, etc. de lo que yá se nos diere anuncia ${ }^{6}$. Pela nuesa parte podríemos camentar qu'estos exemplos, como otros que siguen darréu, podríen entendese dende l'asturianu, lo que sedría un testimoniu inapreciable de lo que debió ser una tendencia al llogru de la cimera coherencia del idioma ${ }^{7}$ llevando la triple distinción xenérica del axetivu (-u/-a/-o), del demostrativu (esti/esta/esto) y del personal (nominativu: el/ella/ello; acusativu: lu/la/lo), tamién al nome que nun lo llograre dafechu anque sí dacuando (cestu/cesta/cesto), y al artículu que, si bien lo algamare $(e l / l a / l o)$, el correspondiente lo nun podía preceder al nome. Nesa mesma llinia podríen citase otros exemplos como:

(5) desde el nogal dicho hasta todo lo arco de aquella costera [1139]

(6) todo lo reburdio valle de so la fuente [754]

(7) Y lo argomal de hacia lo de Pedro Martín, cada y cuando estará seguro [1130]

Pero, al empar, ye verdá qu'esti razonamientu podría tar equivocáu dafechu darréu que la mesma construcción tamién apaez con un nome como cuesta qu'ufierta una terminación asociada davezu al femenín (en -a):

(8) Aquí párate y echa los ojos a lo cuesta falda del dicho cueto [1132]

(9) plantes de pumares y perales (...) todo lo cuesta y lo seco del pradico [1136]

La rempuesta, de toes maneres, taría en que los exemplos ufiertaos nun testimonien un procesu zarráu dafechu sinón que la llingua taba empobinada al llogru plenu de la triple distinción xenérica nel artículu delantre del nome anque entál' nel nome non-cuntable nun s'algamaren tolos finxos. Con too ello ¿acábense equí toles posibilidaes interpretatives? ¿Qué otres víes podríen triase a la gueta d'un bon entendimientu de la construcción gramatical citada?

A lo meyor podemos camentar d'otra miente ensin necesidá de dir mui lloñe nes nueses propuestes. Ye posible que les combinaciones del tipu lo cuesta, lo argomal, etc. nun respuendan namás qu'a una continuación d'otres posibles oraciones onde, consabida del interlocutor-llector una determinada

\footnotetext{
5 Asina «el omezio», «lo omeçidio»Fueru d'Uviéu: Isabel Torrente Fernández \& Ana M. Cano GonzÁlez, eds. (1995): Fueru d'Uviéu. Facsímil del manuscritu del sieglu xIII del Archivu Municipal de la Ciudá d'Uviéu. Llectura y entamu Isabel Torrente; estudiu llingüísticu y torna Ana M. ${ }^{a}$ Cano González. Uviéu, ALLA.

6 Ana María Cano «Algunas aportaciones sobre la diacronía del artículo». Filología Románica 7 (1992): 57-74.

7 X.Ll. García Arias, Gramática Histórica de la Lengua Asturiana, Uviéu, Academia de la Llingua Asturiana, 2003, pxs. 137-138.
} 
referencia, ésta nun se conseñe llueu por innecesaria pa la comprensión. Nesi sen les últimes cinco oraciones citaes, por exemplu, tendríamosles como resultáu d'una reducción aclarada pel contestu; asina podríen entendese como resultáu d'otres previes d'ésti o asemeyáu calter:

*(5’) desde el nogal dicho hasta todo lo \{que ta xunto al\} arco de aquella costera [1139]

*(6’) todo lo \{que ye de\}reburdio, \{en\} valle de so la fuente [754]

*(7’) Y lo \{que ta de\} argomal de hacia lo de Pedro Martín, cada y cuando estará seguro [1130]

*(8’) Aquí párate y echa los ojos a lo \{que ye\} cuesta, falda del dicho cueto [1132]

*(9') plantes de pumares y perales (...) todo lo \{que ye\} cuesta y lo seco del pradico [1136]

Perposiblemente esta interpretación podría sofitase en construcciones averaes pero daqué más esplícites nel nuesu autor:

(10) poner el carro derecho en lo ya lladera [740]

(11) Y assí en lo de Bellencioso sea el cierro; mas lo de roza si no sale para centeno plantarlo del arbolío menor [1136]

*(12) Lo \{que ye\} cuesta y zerro, de roza (...). Lo demás, hasta el prado (...) bellíssima tierra para escanda trigo, zevada» [1130]

*(13) plantes de pumares y perales (...) todo lo \{que ye $\}$ cuesta y lo \{que ye\} seco del pradico [1136]

En realidá éstes sedríen construcciones llingüísticamente esperables pero que reducen elementos naguando por una mayor condensación del llinguaxe. Nun se taría mui lloñe d'un procesu que se conseña n'otres fasteres del mundu llingüísticu hispánicu onde la combinación indicadora de posesión lo + de + nome propiu (Lo de María) pue reducise a Lo María ${ }^{8}$. Almitiendo la citada posibilidá interpretativa ${ }^{9}$ nun se tranca'l camín a que les construcciones lo cuesta, lo argomal, lo reburdio... foren perfechamente acoyíes pol asturianu como una de les víes espresives que'l sistema permitía y que, de mano, nun paecíen ayenes a la so cadarma. Nada tendría de raro que per esa senda entamare l'asturianu a inxerir usos de nomes non-cuntables pre-

8 Álvaro Galmes de Fuentes «Sobre la partícula son antepuesta a nombres de predios mallorquines». Revista de Filología Española 39 (1955), págs. 147 ss.

9 En realidá paecen construcciones asemeyaes orixinariamente a otres como: corpiños a lo de señora [235] de entre los cuernos a lo xarifo [867] todo género de medias y calcetas, a lo guapo [857] no ha de vestir a lo señor. O como dizen a la moda y usos nuevos [550] 
cedíos d'artículu lo. Pero, anque exemplos como lo valle daríen a entender que s'avanzaba nel procesu, ésti quedó tarazáu al dase llueu una castellanización cada vez más fonda del asturianu. Poro, los exemplos de Pumarada abúltennos un bon encontu documental que fadría falta probar con nueves aportaciones si ye que se dieren nos nuesos escritores. 